Systemic lupus erythematosus (SLE) is also associated with smoking. A strong and specific connection of current smoking and $>10$ pack-years of smoking with dsDNA+ SLE has been observed. In patients with SLE, smoking exposure has deleterious effects on lupus morbidity and is related to cumulative chronic damage. In mucocutaneous SLE, smoking have a negative impact on the efficacy of belimumab and a 2 -fold decrease in the proportion of patients achieving cutaneous improvement with antimalarials.

Published data indicate that smoking has a dose-dependent impact on structural damage progression in Ankylosing Spondylitis.

In a large UK cohort, smoking was positively associated with the risk of psoriatic arthritis in the general population, but negatively associated among patients with psoriasis. In addition, smokers with psoriatic arthritis had a poorer response to TNFi's compared to non-smokers.

Regarding osteoporosis, smoking has been identified as a predictor of ten-year fracture risk in men and women, largely independent of the densitometric results. This finding has eventually lead to incorporation of this risk factor into FRAX ${ }^{\circledR}$. The impact of smoking on bone status is mainly associated with the number of smoking years.

In a few reports, it has been observed an association between smoking and carpal tunnel syndrome and inflammatory bowel disease. Finally, current smoking in patients with fibromyalgia is associated with greater pain, possibly as a function of depression.

Disclosure of Interests: Antonio Naranjo Grant/research support from: Amgen, Consultant for: UCB, Speakers bureau: Amgen, UCB

DOI: 10.1136/annrheumdis-2019-eular.8546

\section{SP0089 CONSEQUENCES OF SMOKING CESSATION IN RHEUMATOLOGY}

Helen Harris. Western General Hospital, NHS Lothian, Rheumatology, Edinburgh, United Kingdom

Background: Cigarette smoking is a risk factor for many autoimmune and inflammatory conditions including rheumatoid arthritis (RA), psoriasis and psoriatic arthritis ( $\mathrm{PsO} \mathrm{PsA}$ ), is linked with active skin disease in patients with systemic lupus erythematosis (SLE) and poorer outcomes in patients with ankylosing spondylitis (AS) and systemic sclerosis (SSc). In addition there are well established risks of cardiovascular and respiratory diseases and increased risk of at least 13 cancers in patients that smoke [1]. Patients continuing to smoke after RA is diagnosed often fail to respond or require higher doses of immunosuppressive medications [2]. When arthritis is in remission and biologic dose reduction is attempted, patients that continue to smoke are more likely to have a flare of arthritis after dose reduction [3]. Patients that quit smoking can reduce or stop immunosuppressive medications without experiencing a flare [4]. Smokers have higher rates of infection than non-smokers; immunosuppressive therapy has an increased risk of infection and smoking cessation when starting treatment such as methotrexate or anti-TNF inhibitors can reduce the risk of infection [5]. The five top reasons to deliver brief smoking cessation advice to rheumatology patients are: 1. Improve chances of response to therapy. 2. Reduce infective risks of immunosuppressive therapy. 3. Improve success of medication dose reduction. 4. Reduce cardiovascular and respiratory diseases and cancer risks. 5. Reduce mortality.

Objectives: To demonstrate how brief smoking cessation advice can be delivered in rheumatology outpatient clinics and to show the consequences that this has had for patients, the rheumatology team and the wider healthcare system.

Methods: Delivering a brief intervention on smoking cessation by saying to smokers "Do you know the best way to quit? Use Champix (Varenicline) or Nicotine Replacement Therapy" is an evidence based way to motivate a quit attempt that takes 30 seconds to deliver. A 50 minute talk was presented to the rheumatology team of doctors, nurses and allied health professionals explaining this method of delivering brief advice on smoking cessation. We have made brief advice easy to deliver in the rheumatology clinic by creating a pathway for referral of smokers to the community pharmacist and smoking cessation nurse. A questionnaire has been administered to the rheumatology team to explore their attitudes and beliefs about delivering smoking cessation advice in the rheumatology clinic.

Results: The referral figures for the smoking cessation pathway will be presented. The consequences of delivering brief advice on smoking cessation for the patients, rheumatology team and healthcare system will be reported.

Conclusion: This presentation will show that helping rheumatology smokers to quit is achievable and should be considered an essential part of the rheumatology outpatient consultation.

\section{REFERENCES:}

[1] Arnson Y, Shoenfeld $Y$, Amital H. Effects of tobacco smoke on immunity, inflammation and autoimmunity. Journal of Autoimmunity 34 (2010) J258eJ265.
[2] Harris H, Tweedie F, White M, Samson K. How to persuade patients with Rheumatoid Arthritis to quit smoking. J Rheumatol. 2016 Apr;43(4):691-8. doi: 10.3899/jrheum.141368. Epub 2016 Feb 15.

[3] Gibson J, Harris H, McLaren J. Dose reduction of biologic therapies. EULAR OP0161 June 2012

[4] Andersson M, Bergman S et al, for the BARFOT Study Group $\S$ The Effect of Stopping Smoking on Disease Activity in Rheumatoid Arthritis (RA). Data from BARFOT, a Multicenter Study of Early RA. Open Rheumatol J. 2012; 6: 303-309.

[5] Arcavi L, Benowitz NL. Cigarette smoking and infection. Arch Intern Med. 2004 Nov 8;164(20):2206-16.

Disclosure of Interests: None declared

DOI: 10.1136/annrheumdis-2019-eular.8493

\section{SP0090 INTENSIVE SMOKING CESSATION INTERVENTION}

Ida Kristiane Roelsgaard. Rigshospitalet, Copenhagen Center for Arthritis Research (COPECARE), Center for Rheumatology and Spine Diseases, Glostrup, Denmark

Smokers with an inflammatory joint disease (IJD) experience poorer healthrelated quality of life compared to non-smokers with IJD (1). Smoking may exacerbate the symptoms of the disease and cause a reduced response to antirheumatic treatment (2-4). Smoking cessation interventions have traditionally been designed for people without chronic diseases (5). The literature on smoking cessation interventions to people with IJDs is limited.

Initially, this lecture will focus on "state of the art" regarding smoking cessation interventions to people with IJD.

Firstly, results from a systematic review will be presented. Randomised controlled trials (RCT) were included if they tested any form of smoking cessation intervention for adult daily smokers diagnosed with an IJD (6).

Secondly, the lecture will focus on various interventions for smoking cessation e. g. brief advice, behavioural interventions, motivational interviewing and nicotine replacement therapy.

Finally, principles for an ongoing RCTs where daily smokers with rheumatoid arthritis (RA) are randomised to either an intervention group or to a control group will be presented (7). The intervention consists of motivational counselling combined with nicotine replacement therapy. One patient research partner with RA (former smoker) has been involved with all phases of the study.

The above indicate that smoking cessation may positively impact not only clinical outcomes but also patient reported outcomes. However, a limited number of studies on smoking cessation interventions and the effect of smoking cessation on clinical and patient reported outcomes to people with IJD have been published. The knowledge is therefore limited, and health professionals should focus on this subject to proactively support people with IJDs who wish to quit smoking.

\section{REFERENCES}

[1] Bremander A, Jacobsson LT, Bergman S, Haglund E, Lofvendahl S, Petersson IF. Smoking is associated with a worse self-reported health status in patients with psoriatic arthritis: data from a Swedish populationbased cohort. Clinical rheumatology. 2015;34(3):579-83.

[2] Chang K, Yang SM, Kim SH, Han KH, Park SJ, Shin JI. Smoking and rheumatoid arthritis. International journal of molecular sciences. 2014;15 (12):22279-95.

[3] Abhishek A, Butt S, Gadsby K, Zhang W, Deighton CM. Anti-TNF-alpha agents are less effective for the treatment of rheumatoid arthritis in current smokers. Journal of clinical rheumatology : practical reports on rheumatic \& musculoskeletal diseases. 2010;16(1):15-8.

[4] Soderlin MK, Petersson IF, Geborek P. The effect of smoking on response and drug survival in rheumatoid arthritis patients treated with their first antiTNF drug. Scandinavian journal of rheumatology. 2012;41(1):1-9.

[5] Gritz ER, Vidrine DJ, Fingeret MC. Smoking cessation a critical component of medical management in chronic disease populations. American journal of preventive medicine. 2007;33(6 Suppl):S414-22.

[6] Roelsgaard IK, Esbensen BA, Østergaard M, Rollefstad S, Semb AG Christensen $\mathrm{R}$, et al. Smoking cessation intervention for reducing disease activity in chronic autoimmune inflammatory joint diseases. Cochrane Database of Systematic Reviews. 2018(2).

[7] Roelsgaard IK, Thomsen T, Ostergaard M, Christensen R, Hetland ML, Jacobsen $\mathrm{S}$, et al. The effect of an intensive smoking cessation intervention on disease activity in patients with rheumatoid arthritis: study protocol for a randomised controlled trial. Trials. 2017;18(1):570.

Disclosure of Interests: None declared

DOI: 10.1136/annrheumdis-2019-eular.8573 